\title{
AH Cam: An RR Lyr (Double Mode?) Star with Blazhko Effect
}

\author{
Rafael Garrido ${ }^{1}$, Seung-Lee Kim ${ }^{2,3}$, Jonghyuk $\mathrm{Yi}^{2,3}$, Bo-Won Lee ${ }^{2,3}$, \\ Michele Bossi ${ }^{4}$
}

\begin{abstract}
We report here the discovery of a new frequency not belonging to the Blazhko series and suggest an interpretation based on coupling between a radial and a nonradial mode from multicolor observations.
\end{abstract}

New multicolor observations from Korea and Spain show that the frequency solution for AH Cam is well described, as already shown by Smith et al. (1994), by a series of triplets centered at the main frequency $2.71 \mathrm{~d}^{-1}$ and its multiples, separated by the beat frequency at around $1 / 11 \mathrm{~d}^{-1}$. These triplets are symmetric in frequency, but clearly not symmetric in amplitude and are present around each multiple of the main frequency. A DFT of the residuals shows the presence of a new frequency, not belonging to the triplet series, at $5.4 \mathrm{~d}^{-1}$ which could be a reasonable value for a third radial overtone.

In the $T_{\text {eff }}$ and $\log g$ range of the RR Lyr stars, multicolor observations in the Strömgren photometric system can be useful to discriminate modes as described in Garrido, Garcia-Lobo, \& Rodríguez (1990). For radial modes a positive phase difference between $v$ and $y$ is predicted, whereas a negative value is predicted for any $\ell \neq 0$ nonradial mode. The extremely small values of these phase differences allow us to calculate significant values only for the higher power frequencies. We find a positive value for $f_{\mathrm{a}}$, the main radial frequency, and $2 f_{\mathrm{a}}$, and negative values for $f_{0}$, the Blazhko frequency, and $f_{\mathrm{a}}+f_{0}$, the positive interference with the main frequency. This could be an observational indication that the Blazhko effect is a coupling of a radial mode with a nonradial one as theoretically suggested by Van Hoolst, Dziembowski, \& Kawaler (1998).

\section{References}

Garrido, R., Garcia-Lobo, E., \& Rodríguez, E. 1990, A\&A, 234, 262

Smith, H. A., Matthews, J. M., Lee, K. M., Williams, J., Silbermann, N. A., \& Bolte, M. 1994, AJ, 107, 679

Van Hoolst, T., Dziembowski, W. A., \& Kawaler, S. D. 1998, MNRAS, 297, 536

\footnotetext{
${ }^{1}$ Instituto de Astrofisica de Andalucia, CSIC, Apdo. 3004 18080, Granada, Spain

${ }^{2}$ Korea Astronomy Observatory, Taejon, 305-348, Korea

${ }^{3}$ Department of Astronomy, Seoul National University, 151-742, Korea

${ }^{4}$ Osservatorio Astronomico di Brera, Via Bianchi 46, I-23807, Merate LC, Italy
} 